\title{
Evaluation of the conventional and modified aerosol boxes during tracheal intubation in normal and difficult airways: a randomized, crossover, manikin simulation study
}

\author{
Hyerim Kim ${ }^{1}$ - Jee-Eun Chang ${ }^{1}$ - Dae Kon Kim² ${ }^{2}$ Dongwook Won ${ }^{1}$. Jung-Man Lee ${ }^{1} \cdot$ Tae Kyong Kim $^{1,3}$. \\ Seong-Won $\mathrm{Min}^{1,3} \cdot$ Haeun $\mathrm{Lim}^{3} \cdot$ Seoyoung Ma ${ }^{1}$ Jin-Young Hwang ${ }^{1,3}$
}

Received: 4 May 2021 / Accepted: 16 January 2022 / Published online: 20 January 2022

(c) The Author(s), under exclusive licence to Springer Nature B.V. 2022

\begin{abstract}
The aim of this study was to evaluate conventional and modified aerosol boxes in terms of intubation time, first-pass intubation success, and mouth-to-mouth distance between the laryngoscopist and patient during tracheal intubation in simulated patients with normal and difficult airways. Sixteen anesthesiologists performed tracheal intubations with direct laryngoscope or three different videolaryngoscopes (McGRATH MAC videolaryngoscope, C-MAC videolaryngoscope, and Pentax-AWS) without an aerosol box or with a conventional or a modified aerosol boxes in simulated manikins with normal and difficult airways. Intubation time, first-pass intubation success, and mouth-to-mouth distance during tracheal intubation were recorded. Compared to no aerosol box, the use of a conventional aerosol box significantly increased intubation time in both normal and difficult airways (Bonferroni-corrected $P$-value $\left(P_{\text {corrected }}\right)=0.005$ and $P_{\text {corrected }}=0.003$, respectively). Intubation time was significantly shorter with the modified aerosol box than with the conventional one for both normal and difficult airways $\left(P_{\text {corrected }}=0.003\right.$ and $P_{\text {corrected }}=0.011$, respectively). However, no significant differences were found in intubation time between no aerosol box and the modified aerosol box for normal and difficult airways $\left(P_{\text {corrected }}=0.336\right.$ and $P_{\text {corrected }}=0.112$, respectively). The use of conventional or modified aerosol boxes significantly extended the mouth-to-mouth distances compared to not using an aerosol box during tracheal intubation with each laryngoscope (all $P_{\text {corrected }}<0.05$ ), and the distances were not different between the conventional and modified boxes in normal and difficult airways. The use of modified aerosol box did not increase intubation time and could help maintain a distance from the simulated patients with normal and difficult airways.
\end{abstract}

Keywords COVID-19 $\cdot$ Aerosol box $\cdot$ Airway $\cdot$ Modified $\cdot$ Videolaryngoscope

\section{Introduction}

Jin-Young Hwang

mistyblue15@naver.com

1 Department of Anesthesiology and Pain Medicine, SMGSNU Boramae Medical Center, Boramae-ro, Dongjak-gu, Seoul 156-707, Republic of Korea

2 Department of Emergency Medicine, Seoul National University Bundang Hospital, Gyeonggido, Republic of Korea

3 Department of Anesthesiology \& Pain Medicine, College of Medicine, Seoul National University, Seoul, Republic of Korea
The coronavirus 2019 (COVID-19) pandemic, caused by severe acute respiratory syndrome coronavirus 2 (SARS$\mathrm{CoV}-2$ ), is an ongoing worldwide concern. Symptomatic COVID-19 patients need hospitalization and can require airway management and mechanical ventilation. COVID19 spreads mainly through droplets or aerosols from infected persons [1,2]. Therefore, transmission of COVID-19 to healthcare providers is also one of the major concerns in the COVID-19 outbreak [3, 4]. In particular, aerosol-generating procedures, such as bag mask ventilation and tracheal intubation, are high-risk procedures [5, 6]. Even if the patient does not cough, healthcare providers are at risk of an airborne transmission infection because they cannot avoid getting their face close to the patient's airway. Furthermore, 
standard personal protective equipment (PPE) such as gowns, goggles, masks, and gloves may not be available in some situations, or may not adequately protect healthcare providers as a result displaced or slipped masks or goggles during some urgent clinical situations. The density of droplets or aerosol of the patients would be reduced as the distance from the patient increases [7]. Therefore, it would be safer for healthcare providers to maintain a distance from COVID-19 patients during airway management.

The aerosol box, first designed by Dr. Lai Hsien-Yung [8], is one of the equipments for protecting the healthcare providers from cough dispersion of droplets and containing aerosols during airway management and tracheal intubation. It consists of a transparent acryl barrier enclosure to cover the patient's head and shoulders, with two small holes where clinicians insert their arms and manage the airway (Fig. 1). The arm hole is designed to be round and almost fit the clinician's arms to minimize the risk of contamination with droplets or aerosol from the patient. However, small, round arm holes can limit a clinician's arm movement and lead to longer intubation time and a higher risk of hypoxia, particularly in patients with difficult airways. Therefore, we modified it to produce oval-shaped arm holes to allow more free movement of the arms within the aerosol box, and attached plastic arm sleeve protectors to the hole inside the box to prevent exposure to droplets and aerosol from the patient (Fig. 1). We designed it, and entrusted manufacturing to a handicraftsman. This modified aerosol box can allow an increased range of motion in the arms, and airway management may be less limited than that when using the conventional aerosol box.

In the present study, we evaluated conventional and modified aerosol boxes in terms of intubation time, first-pass intubation success, and mouth-to-mouth distance between the laryngoscopist and patient during tracheal intubation in simulated patients with normal and difficult airways.

\section{Methods}

This study was a randomized, crossover, manikin simulation study. This study was approved by the Institutional Review Board of our hospital (no. 10-2021-11). Written informed consent was obtained from all anesthesiologists who participated in this study.

A conventional aerosol box was manufactured in the form of an early generation transparent acryl aerosol box with two round arm holes, as described by Dr. Lai Hsien-Yung [8]. The diameter of each arm hole was $10 \mathrm{~cm}$. A modified aerosol box was developed with oval-shaped arm holes and a $50-\mathrm{cm}$ plastic arm sleeve protector attached to each arm hole. The diameter of each arm hole was $15 \mathrm{~cm}$ at the horizontal axis and $20 \mathrm{~cm}$ at the vertical axis (Fig. 1).

Eighteen board-certified staff anesthesiologists, experienced in performing tracheal intubation (more than 500 intubations using direct laryngoscope and 100 intubations using videolaryngoscopes), were included. Participants wore PPE (mask, goggle, gown, and gloves) in accordance with the institutional guidelines. Gown and goggle were reused in this study to save them. Each participant performed 24 tracheal intubations using direct laryngoscope or three different videolaryngoscopes without an aerosol box or with the conventional or modified aerosol boxes in normal and difficult airway manikins (Laerdal Airway Management Trainer and Deluxe Difficult Airway Trainer [Laerdal Medical Corp., NY, USA], respectively). With the difficult airway model, the tongue was inflated to achieve a Cormack-Lehane grade 3 under direct laryngoscopy, which was verified by

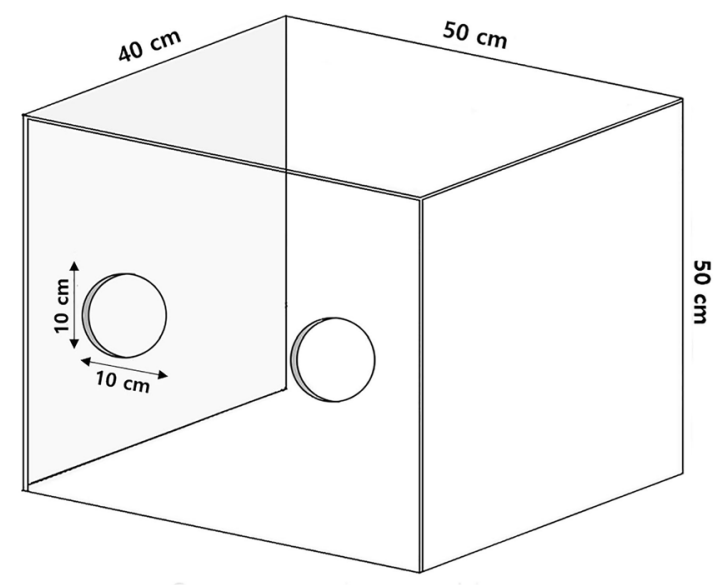

Conventional aerosol box

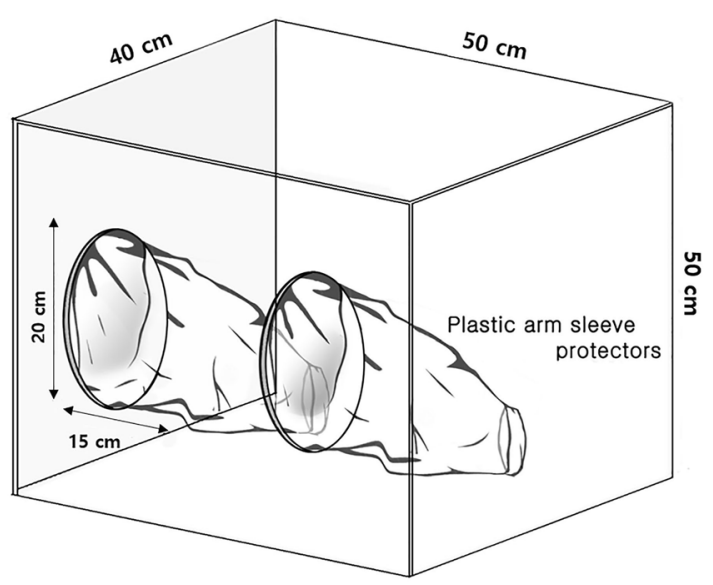

Modified aerosol box

Fig. 1 Conventional and modified aerosol boxes 
an independent staff anesthesiologist not involved in the study. The videolaryngoscopes used in this study were McGRATH MAC videolaryngoscope (Covidien, Medtronic Inc., Dublin, Ireland), C-MAC videolaryngoscope (Karl Storz Endoscopy, Inc., Tuttlingen, Germany), and PentaxAWS (Airway Scope; Hoya Corporation, Tokyo, Japan). A malleable stylet was used during tracheal intubation except for with the Pentax-AWS. Participants were instructed to use each videolaryngoscope and aerosol box and allowed to practice tracheal intubation using them in the manikins before simulation. The order of tracheal intubations was randomized using a computer-generated program, and participants were blinded to the order. The operating table was supine at the same level as the participant's anterior superior iliac crest, and its height was modified if the participant requested. When the tracheal tube with an internal diameter of $7.5 \mathrm{~mm}$ was advanced past the vocal cords, an assistant removed the stylet, and the tracheal tube was advanced into the trachea. The assistant then inflated the tracheal tube cuff and connected the tube connector to a resuscitation bag to inflate the lungs and check whether tracheal intubation was successful. Intubation time was defined as the time from picking up the direct laryngoscope or videolaryngoscope to confirmed successful intubation with the first lung inflation. If tracheal intubation was not performed within $180 \mathrm{~s}$ or esophageal intubation occurred, it was recorded as a failure. A camera was installed parallel to the operating bed, and another assistant took a picture while participants inserted the tracheal tube into the vocal cords. The mouth-to-mouth distance between the laryngoscopist and manikin was measured in the picture. Each participant performed no more than three intubations in each normal and difficult model of the manikin on the same day.

The primary outcome was the difference in intubation time between conventional and modified aerosol boxes. Secondary outcomes included the success rate of the first-pass intubation and the mouth-to-mouth distance during tracheal intubation among the use and type of aerosol box, and the mouth-to-mouth distance among direct laryngoscope and different videolaryngoscopes.

Sample size was calculated based on a preliminary study showing a intubation time of $41.5 \pm 51.1$ (mean \pm SD) sec during tracheal intubation using direct laryngoscopy between conventional and modified aerosol boxes in a simulated patient with a difficult airway. A minimum of 14 samples were required to achieve $80 \%$ power and a significance level of $\alpha=0.05$. Considering the potential dropout rate, 18 participants were included.

SPSS version 26 for Windows (IBM, Armonk, NY, USA) was used for the statistical analyses. Normality of data distribution was tested using the Shapiro-Wilk test. Data are expressed as mean $\pm \mathrm{SD}$, frequency $(\%)$, or mean difference (interval estimates). Repeated measures analysis of variance
(RM-ANOVA) or the Friedman test were used to analyse intubation time and the mouth-to-mouth distance, depending on the use and type of aerosol box (three factors: none, conventional, and modified) and the type of laryngoscopes (four factors: direct laryngoscope, McGRATH MAC videolaryngoscope, C-MAC videolaryngoscope, and Pentax-AWS). If any significant differences were found in the outcomes without a significant interaction between the different factors, the Bonferroni correction was applied for post-hoc comparison. If significant interactions were found between the different factors, a paired t-test or Wilcoxon's signed rank test with Bonferroni correction was performed. Bonferroni correction was applied by multiplying the uncorrected $P$-value by the number of comparisons (i.e. 3 ) for the outcomes among the use and type of aerosol box and by the number of comparisons (i.e. 6) for the outcomes among direct laryngoscope and three different videolaryngoscopes. The McNemar test was used to compare the success rate of first-pass intubation with each laryngoscope among the use and type of aerosol box. A $P$-value $<0.05$ and Bonferroni corrected $P$-value $\left(P_{\text {corrected }}\right)<0.05$ were considered statistically significant.

\section{Results}

Among the 18 anesthesiologists who participated in this study, two did not complete the study; the remaining 16 participants completed the study. All of the participants had recent experiences of more than 70 intubations using three videolaryngoscopes used in this study (at least more than 15 intubations using each of them) when they were enrolled in this study.

Intubation time and first-pass intubation success are presented depending on the use and type of aerosol box and the different types of laryngoscopes in normal and difficult airways (Table 1). The RM-ANOVA revealed no significant interactions between the use and type of aerosol box and the type of laryngoscope in normal $(P=0.546)$ and difficult airways $(P=0.137)$. However, there was a significant difference in intubation time according to the use and type of aerosol box in normal $(P<0.001)$ and difficult airways $(P=0.002)$. Intubation time according to the use and type of aerosol boxes is shown in Table 2. Compared to no aerosol box, the use of a conventional aerosol box significantly increased intubation time for both normal and difficult airways $\left(P_{\text {corrected }}=0.005\right.$ and $P_{\text {corrected }}=0.003$, respectively). The intubation time was significantly shorter with the modified aerosol box than with the conventional box for both normal and difficult airways $\left(P_{\text {corrected }}=0.003\right.$ and $P_{\text {corrected }}=0.011$, respectively). However, no significant differences were found in intubation time between no aerosol box and the modified box for normal and difficult airways $\left(P_{\text {corrected }}=0.336\right.$ and $P_{\text {corrected }}=0.112$, respectively $)$. The 
Table 1 Intubation time, and first-pass intubation success during tracheal intubation without an aerosol box and with conventional and modified aerosol boxes in normal and difficult airways

\begin{tabular}{|c|c|c|c|c|c|c|}
\hline & \multicolumn{3}{|c|}{ Normal airway } & \multicolumn{3}{|c|}{ Difficult airway } \\
\hline & None & Conventional & Modified & None & Conventional & Modified \\
\hline \multicolumn{7}{|l|}{ Direct laryngoscope } \\
\hline Intubation time; sec & $13.6 \pm 2.1$ & $17.0 \pm 4.3$ & $14.4 \pm 2.3$ & $20.8 \pm 6.3$ & $67.8 \pm 60.5$ & $26.8 \pm 10.6$ \\
\hline $\begin{array}{l}\text { First-pass success; \% } \\
\text { McGRATH MAC }\end{array}$ & 100 & 100 & 100 & 100 & 100 & 100 \\
\hline Intubation time; sec & $13.2 \pm 3.3$ & $17.6 \pm 10.4$ & $13.9 \pm 4.7$ & $18.7 \pm 7.7$ & $22.8 \pm 10.8$ & $19.5 \pm 7.7$ \\
\hline $\begin{array}{l}\text { First-pass success; \% } \\
C-M A C\end{array}$ & 100 & 100 & 100 & 100 & 100 & 100 \\
\hline Intubation time; sec & $14.0 \pm 3.9$ & $17.2 \pm 4.4$ & $15.9 \pm 3.9$ & $19.4 \pm 5.2$ & $20.7 \pm 5.9$ & $19.3 \pm 5.1$ \\
\hline $\begin{array}{l}\text { First-pass success; \% } \\
\text { Pentax-AWS }\end{array}$ & 100 & 100 & 100 & 100 & 100 & 100 \\
\hline Intubation time; sec & $11.1 \pm 4.0$ & $11.7 \pm 3.0$ & $11.2 \pm 3.0$ & $12.4 \pm 2.5$ & $14.3 \pm 3.4$ & $13.3 \pm 4.2$ \\
\hline First-pass success; $\%$ & 100 & 100 & 100 & 100 & 100 & 100 \\
\hline
\end{tabular}

Values are presented as mean $\pm \mathrm{SD}$ or percentage

\begin{tabular}{llllll}
\hline Comparison & Normal airway & & & Difficult airway & \\
\cline { 2 - 3 } \cline { 6 - 7 } & Difference (s) & $P_{\text {corrected }}$ & & Difference (s) & $P_{\text {corrected }}$ \\
\hline None vs. Conventional & $-2.9(-4.9$ to -0.9$)$ & 0.005 & & $-13.6(-22.4$ to -4.8$)$ & 0.003 \\
None vs. Modified & $-0.9(-2.3$ to 0.5$)$ & 0.336 & & $-1.9(-4.2$ to 0.3$)$ & 0.112 \\
Conventional vs. Modified & $2.0(0.7$ to 3.3$)$ & 0.003 & & $11.7(2.5$ to 20.9$)$ & 0.011 \\
\hline
\end{tabular}

Values are presented as mean difference (95\% confidence interval)

$P_{\text {corrected, }}$ Bonferroni-corrected $P$-value
Table 2 Intubation time depending on the use and type of aerosol box in normal and difficult airways first-pass intubation success was $100 \%$ irrespective of the use and type of aerosol box and the type of laryngoscope in normal and difficult airways.

The mouth-to-mouth distances between the laryngoscopist and patient with different laryngoscopes and aerosol boxes are presented in Table 3. The RM-ANOVA revealed significant interactions between the use and type of aerosol box and the type of laryngoscope for normal $(P<0.001)$ and difficult airways $(P<0.001)$. Significant differences were also observed in the mouth-to-mouth distance based on the use and type of aerosol box $(P<0.001)$ and type of laryngoscope $(P<0.001)$ for both normal and difficult airways. The use of a conventional or modified aerosol box significantly extended the mouth-to-mouth distance compared with that with no use of an aerosol box during tracheal intubation with each laryngoscope (all $P_{\text {corrected }}<0.05$ ); the distance was not different between the conventional and modified boxes for normal and difficult airways. Without an aerosol box, the mouth-to-mouth distance was significantly larger during tracheal intubation with each videolaryngoscope compared with that with direct laryngoscopy for normal and difficult airways (all $P_{\text {corrected }}<0.05$ ). With the use of either conventional or modified aerosol boxes, the C-MAC videolaryngoscope significantly increased the mouth-tomouth distance compared to that with direct laryngoscope,
Table 3 Mouth-to-mouth distance $(\mathrm{cm})$ during tracheal intubation with direct laryngoscope and three different videolaryngoscopes depending on the use and type of aerosol box in simulated patients with normal and difficult airways

\begin{tabular}{llll}
\hline & \multicolumn{2}{l}{ Aerosol box } & \\
\cline { 2 - 4 } & None & Conventional & Modified \\
\hline Normal airway & & & \\
Direct laryngoscope & $22.0 \pm 8.5$ & $33.8 \pm 4.2^{\mathrm{a}} \mathrm{c}$ & $33.1 \pm 4.7^{\mathrm{ac}}$ \\
McGRATH MAC & $29.0 \pm 3.6^{\mathrm{b}}$ & $35.4 \pm 3.7^{\mathrm{ac}}$ & $34.1 \pm 4.8^{\mathrm{a} \mathrm{c}}$ \\
C-MAC & $32.4 \pm 4.3^{\mathrm{b}}$ & $38.8 \pm 3.3^{\mathrm{a}}$ & $38.9 \pm 3.7^{\mathrm{a}}$ \\
Pentax-AWS & $31.9 \pm 3.7^{\mathrm{b}}$ & $36.5 \pm 4.1^{\mathrm{ac}}$ & $34.6 \pm 2.6^{\mathrm{a} \mathrm{c}}$ \\
Difficult airway & & & \\
Direct laryngoscope & $19.1 \pm 7.0$ & $32.4 \pm 4.9^{\mathrm{a}} \mathrm{c}$ & $30.2 \pm 6.4^{\mathrm{ac}}$ \\
McGRATH MAC & $27.4 \pm 5.4^{\mathrm{b}}$ & $34.1 \pm 2.6^{\mathrm{ac}}$ & $32.8 \pm 3.8^{\mathrm{ac}}$ \\
C-MAC & $31.7 \pm 2.9^{\mathrm{b}}$ & $39.1 \pm 3.0^{\mathrm{a}}$ & $38.0 \pm 3.1^{\mathrm{a}}$ \\
Pentax-AWS & $30.8 \pm 2.6^{\mathrm{b}}$ & $34.0 \pm 3.6^{\mathrm{ac}}$ & $32.8 \pm 3.1^{\mathrm{ac}}$ \\
\hline
\end{tabular}

Values are presented as mean \pm SD. $P_{\text {corrected, }}$ Bonferroni-corrected $P$-value

${ }^{\mathrm{a}} P_{\text {corrected }}<0.05$ vs. no box

${ }^{\mathrm{b}} P_{\text {corrected }}<0.05$ vs. direct laryngoscope (without aerosol box)

${ }^{\mathrm{c}} P_{\text {corrected }}<0.05$ vs. C-MAC (using either conventional or modified aerosol boxes) 
McGRATH MAC videolaryngoscope, and Pentax-AWS for both normal and difficult airways (all $P_{\text {corrected }}<0.05$ ).

\section{Discussion}

This study showed that the use of a modified aerosol box leads to a shorter intubation time compared to that when using a conventional box, and no significant difference was observed in intubation time between no use of aerosol box and the modified box for normal and difficult airways. Furthermore, the mouth-to-mouth distance between the laryngoscopist and patient was significantly increased with the use of a conventional or modified aerosol box, and the mouth-to-mouth distance did not differ between the two different aerosol boxes for both normal and difficult airways. When conventional or modified aerosol boxes were used, the mouth-to-mouth distance was significantly greater with the C-MAC videolaryngoscope than that with direct laryngoscope, McGRATH MAC videolaryngoscope, or Pentax-AWS.

The aerosol box can be easily fabricated and may help protect healthcare providers during airway management. The early generation aerosol box incorporates two arm holes where the clinician's hands are inserted to perform the airway procedures, and the movement of arms can be limited because of the tight size of each arm hole. Our finding that the use of a conventional aerosol box increased intubation time was consistent with a previous study evaluating the effect of early generation aerosol box on tracheal intubation with a videolaryngoscope [9]. The modified aerosol box had larger oval-shaped arm holes and plastic arm sleeve protectors attached to the holes. Thus, the movement of arms are less impeded during airway management. In the present study, the intubation time with the modified aerosol box was significantly shorter than that with the conventional one, and not different from that when not using an aerosol box for normal and difficult airways. Therefore, the use of a modified aerosol box would protect healthcare providers without delaying intubation time.

In the present study, the difference in intubation time between conventional and modified aerosol boxes might be considered clinically insignificant for a normal airway. However, we found that the difference in intubation time was increased between the two different aerosol boxes for a difficult airway. Furthermore, intubation time was not different when performing tracheal intubation with no use of the aerosol box or when using the modified box for a difficult airway. Compared to normal airways, more attempts and airway maneuvers are required for difficult airway management; thus, apnea time and subsequent risk of hypoxia are increased. In this respect, the modified aerosol box may be useful particularly for managing difficult airways, and the clinical significance of our findings cannot be ignored.

During airway management, maintaining a distance from the COVID-19 patient may be a strategy to protect healthcare providers, regardless of them wearing PPE [7]. In the present study, the use of either of the two different aerosol boxes increased the mouth-to-mouth distance between the participant and patient during tracheal intubation using direct laryngoscope or three different videolaryngoscopes. Without an aerosol box, clinicians may tend to get closer to the patient's mouth during tracheal intubation. The presence of an aerosol box can force them to maintain their distance from patients.

According to the consensus guidelines for airway management in patients with COVID-19 [10], videolaryngoscopy is recommended to facilitate the laryngeal view and maintain distance from the patients relative to direct laryngoscopy. Currently, various types of videolaryngoscopes have been introduced, and a videolaryngoscope that can be used as far away as possible from the patient airway would be safer for healthcare workers providing airway management to COVID-19 patients. In the present study, when an aerosol box was not applied, the use of videolaryngoscopes extended the mouth-to-mouth distance compared to the use of direct laryngoscope, which was in line with a previous report where showed that videolaryngoscopy increased the mouth-to-mouth distance compared with direct laryngoscopy [11]. When using the aerosol boxes, the C-MAC videolaryngoscope provided a larger mouth-to-mouth distance than direct laryngoscopy and other videolaryngoscopes, and the mouth-to-mouth distance did not differ between the direct laryngoscope, McGRATH MAC videolaryngoscope, and Pentax-AWS. While McGRATH MAC and Pentax-AWS have screens on the top of the laryngoscopes, the C-MAC videolaryngoscope has a stand screen separate from the laryngoscope. Therefore, clinicians can watch airway structures on a separate screen at a greater distance. In principle, reusable standard and videolaryngoscopes should undergo high-level sterilization or decontamination in the management of COVID-19 patients. The C-MAC has an advantage in terms of sterilization because its electronic module and handle can undergo high-level sterilization or decontamination contrary to other videolaryngoscopes [12].

During tracheal intubation, removal of a malleable stylet by an assistant may provoke scattering of secretions or droplets, which may increase the risk of transmission with or without an aerosol box. Pentax-AWS has a channeled blade, unlike the direct laryngoscope and other videolaryngoscopes used in this study. A tracheal tube was attached to the channel on the right side of the blade without the stylet before the insertion of the Pentax-AWS. Therefore, assistance for removing the stylet was not required, and the risk of scattering droplets or aerosols may be reduced with the 
Pentax-AWS, although the effect of different videolaryngoscopes on the spread of droplets or aerosols during tracheal intubation was not evaluated in this study.

In the present study, we focused on intubation time, firstpass intubation success, and the mouth-to-mouth distance with the use of an aerosol box. However, several concerns about the use of an aerosol box, such as airborne particle exposure related to the structure of an aerosol box and its effectiveness of containing aerosols during aerosol-generating procedures, have been raised [13-15]. Aerosol boxes may disturb airway management, particularly with some airway adjuncts (i.e. tracheal introducers), in difficult airways, or during airway emergencies [16]. Furthermore, environmental contamination through the access holes, disruption of PPE, and secondary aerosolization upon removal or cleaning can occur with the use of aerosol boxes [16, 17]. In the present study, airway management was simulated inside the aerosol boxes in full PPE because aerosol boxes cannot completely protect healthcare providers [17]. Aerosol boxes should never substitute proficient and adequate PPE. Systematic preparedness, planning, and centralization of airway resources are also suggested as an important strategy for safe airway management in COVID-19 patients [18]. Further studies about evaluating those concerns and developing more effective protective strategies for the staff and patients are required.

This study had several limitations. First, participants who performed tracheal intubation and assessors could not be blinded to the use and type of aerosol box and type of laryngoscope. However, they followed a standardised protocol. Second, the effect of different aerosol boxes and different laryngoscopes on viral exposure and contamination were not evaluated. Third, this study was performed in simulated manikins with normal and difficult airways in a well-prepared operating room, and a difficult airway was simulated by inflating the tongue to achieve a high Cormack-Lehane grade. However, there are various factors contributing to a difficult airway, such as obesity or head and neck malignancies. Moreover, our results may not reflect difficult airway managemet in the actual clinical practice.

In conclusion, the use of modified aerosol box did not increase intubation time compared to no use of an aerosol box, and could help maintain a distance from the simulated patients with normal and difficult airways.

\section{References}

1. Wang W, Xu Y, Gao R, Lu R, Han K, Wu G, et al. Detection of SARS-CoV-2 in different types of clinical specimens. JAMA. 2020;323:1843-4.

2. van Doremalen N, Bushmaker T, Morris DH, Holbrook MG, Gamble A, Williamson BN, et al. Aerosol and surface stability of SARS-CoV-2 as compared with SARS-CoV-1. N Engl J Med. 2020;382:1564-7.

3. Wilson NM, Norton A, Young FP, Collins DW. Airborne transmission of severe acute respiratory syndrome coronavirus-2 to healthcare workers: a narrative review. Anaesthesia. 2020;75:1086-95.

4. El-Boghdadly K, Wong DJN, Owen R, Neuman MD, Pocock S, Carlisle JB, et al. Risks to healthcare workers following tracheal intubation of patients with COVID-19: a prospective international multicentre cohort study. Anaesthesia. 2020;75:1437-47.

5. Tran K, Cimon K, Severn M, Pessoa-Silva CL, Conly J. Aerosol generating procedures and risk of transmission of acute respiratory infections to healthcare workers: a systematic review. PLoS ONE. 2012;7:e35797.

6. Jackson T, Deibert D, Wyatt G, Durand-Moreau Q, Adisesh A, Khunti K, et al. Classification of aerosol-generating procedures: a rapid systematic review. BMJ Open Respir Res. 2020;7:e000730.

7. Chao CYH, Wan MP, Morawska L, Johnson GR, Ristovski ZD, Hargreaves M, et al. Characterization of expiration air jets and droplet size distributions immediately at the mouth opening. J Aerosol Sci. 2009;40:122-33.

8. Everington K. Taiwanese doctor invents device to protect US doctors against coronavirus. Taiwan News. https://www.taiwannews. com.tw/en/news/3902435.

9. Begley JL, Lavery KE, Nickson CP, Brewster DJ. The aerosol box for intubation in coronavirus disease 2019 patients: an in-situ simulation crossover study. Anaesthesia. 2020;75:1014-21.

10. Cook TM, El-Boghdadly K, McGuire B, McNarry AF, Patel A, Higgs A. Consensus guidelines for managing the airway in patients with COVID-19: Guidelines from the Difficult Airway Society, the Association of Anaesthetists the Intensive Care Society, the Faculty of Intensive Care Medicine and the Royal College of Anaesthetists. Anaesthesia. 2020;75:785-99.

11. Hall D, Steel A, Heij R, Eley A, Young P. Videolaryngoscopy increases "mouth-to-mouth" distance compared with direct laryngoscopy. Anaesthesia. 2020;75:822-3.

12. Bowdle A, Jelacic S, Shishido S, Munoz-Price LS. Infection prevention precautions for routine anesthesia care during the SARSCoV-2 pandemic. Anesth Analg. 2020;131:1342-54.

13. Dalli J, Khan MF, Marsh B, Nolan K, Cahill RA. Evaluating intubation boxes for airway management. $\mathrm{Br} \mathrm{J}$ Anaesth. 2020;125:e293-5.

14. Kearsley R. Intubation boxes for managing the airway in patients with COVID-19. Anaesthesia. 2020;75:969.

15. Simpson JP, Wong DN, Verco L, Carter R, Dzidowski M, Chan PY. Measurement of airborne particle exposure during simulated tracheal intubation using various proposed aerosol containment devices during the COVID-19 pandemic. Anaesthesia. 2020;75:1587-95.

16. Sorbello M, Rosenblatt W, Hofmeyr R, Greif R, Urdaneta F. Aerosol boxes and barrier enclosures for airway management in COVID-19 patients: a scoping review and narrative synthesis. $\mathrm{Br}$ J Anaesth. 2020;125:880-94.

17. Hofmeyr R, Sorbello M. Airway management in covid-19: time to start thinking outside the box? S Afr J Anaesth Analg. 2021;27:4-11.

18. Sorbello M, Morello G, Pintaudi S, Cataldo R. COVID-19: intubation kit, intubation team, or intubation spots? Anesth Analg. 2020;131:e128-30.

Publisher's Note Springer Nature remains neutral with regard to jurisdictional claims in published maps and institutional affiliations. 